ESAIM: COCV 22 (2016) 939-952

DOI: $10.1051 / \mathrm{cocv} / 2016048$
ESAIM: Control, Optimisation and Calculus of Variations

www.esaim-cocv.org

\title{
CRITICAL POINTS AT INFINITY IN YAMABE CHANGING-SIGN EQUATIONS *
}

\begin{abstract}
ABBAS BAHRI**
Abstract. In the well-known paper [A. Bahri and J.M. Coron, Commun. Pure Appl. Math. 41 (1988) 253-294], Bahri and Coron develop the theory of critical points at innity and find the solutions of Yamabe problem via Morse theory. This is a very delicate problem because of the lack of compactness caused by the invariance under the conformal group. To obtain the desired results, one needs a careful analysis on the change of the topology of the level sets. In this work, the author continues to use these ideas and give a preliminary study of the topological features for the Yamabe sign-changing variational problem on domains of $\mathbb{R}^{3}$ or on spheres $\mathbb{S}^{3}$. One of key points consists to understand the Morse relations at innity based on the expansion of the energy functional in a neighborhood of innity. In particular, one study weather the relation $\partial^{\infty} \circ \partial^{\infty}=0$ holds where $\partial^{\infty}$ is the intersection operator at innity. Although I could not understand completely the details, I believe such study is very delicate and the ideas and techniques developed could be also useful in the others context, in particular, some conformal invariant problems like Yang-Mills equations and harmonic maps. I recommend strongly the publication of the paper.
\end{abstract}

Mathematics Subject Classification. 35B38, 37B30.

Received June 6, 2016. Revised June 7, 2016.

\section{INTRODUCTION}

The present paper falls within the framework of the theory of critical points at infinity, initiated in 1978 in the field of Contact Form Geometry and which we developed, together with my collaborator Coron, over the years 1984-1986 on Yamabe-type equations and on scalar curvature problems, [1-5] and more.

The ideas which are presented here can be easily connected to our joint paper with J.M. Coron [4], despite the improved interpretations and the increase in power in the tools.

This paper therefore may be seen as an application of the ideas of [4] to Yamabe Changing-Sign equations.

The main result involves an understanding of the Morse relations at infinity for Yamabe changing-sign problems, on $S^{3}$ or on a domain $\Omega$ of $\mathbb{R}^{3}$. We study in particular (Part III) whether the relation $\partial^{\infty} \circ \partial^{\infty}=0$ holds. $\partial^{\infty}$ is the intersection operator at infinity for the Yamabe changing sign-problem.

This study relies on a Morse lemma at infinity established in [6], under some conditions which are to be removed for the general theory to proceed. This expansion (Part II) should be useful in other problems such as Yang-Mills equations in dimension 4, the harmonic map problem in dimension 2 etc.

Keywords and phrases. Critical points, Yamabe equation, sign-changing solutions.

* For my collaborator J.M.Coron, on his sixtieth birthday

1 Department of Mathematics, Rutgers University - Hill Center for the Mathematical Sciences, 110 Frelinghuysen Rd., Piscataway, NJ 08854-8019, USA. karine.beauchard@ens-rennes.fr

** Deceased. 
After recalling in details the expansion at infinity of [6] and after studying of the Morse relation $\partial^{\infty} \circ \partial^{\infty}=$ 0 , we also extend in the present paper the so-called "Bahri-Coron" topological argument to the framework of Yamabe changing-sign problems. We find conditions for this extension which might appear at first glance to be stringent. But they can be weakened; they might also be verified for some subset of critical points at infinity in this variational problem. This is Part IV of this paper.

The hope that these results will be helpful for the finding of infinitely many solutions to the equation $\Delta u+u^{5}=0$ in $\Omega, u_{\mid \partial \Omega}=0$, where $\Omega$ a non-contractible bounded and regular domain of $\mathbb{R}^{3}$.

On a personal note and as a conclusion to this brief introduction, we hope that Jean-Michel will see in the present paper, written for his sixtieth birthday, the expression of our gratitude for a creative collaboration!

\section{Part I: The Relation $\partial \circ \partial=0$ in A Given VARIATIONAL PROBlem}

We consider in the sequel a variational problem and its intersection operator $\partial$. The relationship $\partial \circ \partial=0$ holds for this variational problem once a Morse-Smale pseudo-gradient is defined. We assume that the PalaisSmale condition and all transversality conditions are verified for this variational problem, which is equipped with the pseudo-gradient we are referring to. If required, we can extend the Morse Theory with the addition of the appropriate critical points at infinity so that the Palais-Samle condition, also appropriately extended to include the critical points at infinity, holds.

There is a nearly geometric representation of the relation $\partial \circ \partial=0$ : taking a dominating critical point $x_{k+2}$, of index $(k+2)$, and a dominated critical point at infinity $x_{k}$, of index $k$, we assume, for simplicity, that the Morse relations of order $m$, that is the Morse relations involving a critical point of index $m x_{m}$ with a critical point of index $(m-1) x_{m-1}$, are all of algebraic numbers equal either to 1 or to -1 or to 0 and we assume that this algebraic number corresponds to the actual number of flow-lines (with a sign assigned to them according to orientation) from $x_{m}$ to $x_{m-1}$.

Then the relation $\partial \circ \partial=0$, between $x_{k+2}$ and $x_{k}$, may be viewed as represented by kites:

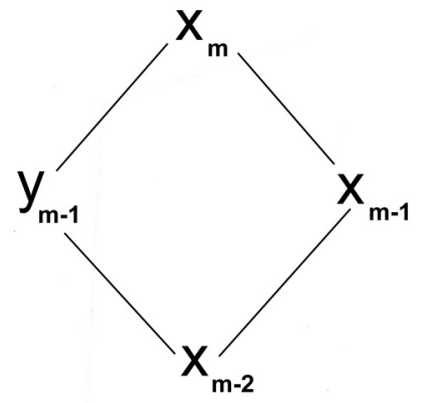

\section{left side of the kite}

\section{right side of the kite}

The algebraic intersection number of each branch of the kite above $z_{l}-z_{l-1}, z_{l}=x_{m}, x_{m-1}$ or $y_{m-1}$, $z_{l-1}=x_{m-1}, y_{m-1}$ or $x_{m-2}$ is \pm 1 . The products of the signs attributed to the two branches on each side of a kite are opposite in sign.

There can be several kites involving two critical points $x_{k+2}$ and $x_{k}$; but all critical points dominated by $x_{k+2}$ and dominating $x_{k}$ are involved in exactly one kite.

When the intersection numbers are not 0,1 or -1 or when there is multiplicity of flow-lines, we can break the multiplicity after perturbation of the variational problem. We can also complete cancellations of flow-lines of opposite intersection numbers and the result holds again.

This provides a nice and simple geometric representation for the relation $\partial \circ \partial=0$. 


\section{Part II: The Yamabe sign-Changing Variational Problem, Expansion on S $^{3}$}

The variational problem corresponding to the Yamabe Changing-Sign equations has been studied in [6]. An expansion at infinity, under some additional assumptions for which we refer to [6], has been derived. The $\omega_{i} \mathrm{~S}$ below are the variational solutions of the Yamabe changing-sign problem on $\mathbb{R}^{3}$, the $a_{i}$ s are their concentration points, the $\lambda_{i}$ s their concentrations. These data may be viewed indifferently on $S^{3}$ or on $\mathbb{R}^{3}$, see [6] for more details on these definitions etc.

Functions $u$ that are in a neighborhood of infinity read:

$$
u=\Sigma \alpha_{i} \omega_{i}+v
$$

where $v$ is small in the " $H^{1 "}$-norm and satisfies various orthogonality conditions, see below.

The functional

$$
J(u)=\frac{\left(\int|\nabla u|^{2} \mathrm{~d} x\right)^{3}}{\int u^{6} \mathrm{~d} x}
$$

then expands into:

$$
J\left(\sum_{i=1}^{p} \alpha_{i} \omega_{i}+v\right)=\frac{\left(\sum_{i=1}^{p} \tilde{\alpha}_{i}^{2} \int\left|\nabla \bar{\omega}_{i}\right|^{2} \mathrm{~d} x\right)^{3}}{\left(\sum_{i=1}^{p} \tilde{\alpha}_{i}^{6} \int \bar{\omega}_{i}^{6} \mathrm{~d} x\right)} \times\left[1-\bar{c} \sum_{i \neq j} \bar{\omega}_{i}\left(\tilde{a}_{j}\right) \bar{\omega}_{j}^{\infty} \tilde{\epsilon}_{i j}-\sum_{i \neq j} c_{i j}\left(\bar{\omega}_{i}, \bar{\omega}_{j}\right) \tilde{\epsilon}_{i j}^{3}+Q(V, V)\right] .
$$

The expansion, just as a usual Morse Lemma does, see e.g. [6], involves an invertible change of variables. Over this change of variables, the variables $\left(\alpha_{i}, a_{i}, \lambda_{i}, v\right)$ associated to the representation of $u$ are changed into $\left(\tilde{\alpha}_{i}, \tilde{a}_{i}, \tilde{\lambda}_{i}, V\right) \cdot \bar{\omega}_{i}$ is a rescaled version of $\omega_{i}$, with concentration $\lambda=1$ for example and $a_{i}$ is at the North pole of $S^{3}$. The concentration points $a_{i}$ are changed into $\tilde{a}_{i}$, the concentrations $\lambda_{i} \mathrm{~s}$ are changed into $\tilde{\lambda}_{i} \mathrm{~s}$ and the $\epsilon_{i j} \mathrm{~s}$, with

$$
\epsilon_{i j}=\frac{1}{\left(\frac{\lambda_{i}}{\lambda_{j}}+\frac{\lambda_{j}}{\lambda_{i}}+\lambda_{i} \lambda_{j}\left|a_{i}-a_{j}\right|^{2}\right)^{1 / 2}}
$$

are changed into their corresponding values with the new variables. $\bar{\omega}_{j}^{\infty}$ is the value of $\bar{\omega}_{j}$ at the south pole of $S^{3} \cdot c_{i j}\left(\bar{\omega}_{i}, \bar{\omega}_{j}\right)$ is a positive constant depending on the rescaled versions of $\omega_{i}, \omega_{j}$, that is depending on $\bar{\omega}_{i}$ and $\bar{\omega}_{j}$. Implicit in the definition of these is a parameter of relative rotation $\sigma$ in $O(3)$ that tracks the relative position of $\nabla \omega_{i}$ with respect to $\nabla \omega_{j}$ at the south or north pole of $S^{3}$.

The Morse lemma is established in [6] under various "reasonable" assumptions, see [6]. We have conjectured in [6] that it holds in full generality and we assume in this paper that it does.

The proof of [6] uses several constructions of local pseudo-gradients for the functional. These constructions are assumed to be known in this paper; we refer the reader to [6] for them.

Extension to domains of $\mathbb{R}^{3}$ of this expansion can be "guessed", with the use of the regular part of the Green's function on these domains for the Dirichlet problem, as in [4,5]. This "guess" should be transformed, of course, into a rigorous proof. The observations below assume that this transformation has been completed: we will use the "natural" expansion of the Yamabe changing-sign problem that we can "guess" from the expansion above.

The third part of this paper is devoted to:

\section{PART III: A FEW observations About The Topology AT Infinity using the ABove EXPANSION}

The above expansion shows that the variational problem at infinity can be split into two largely independent other variational problems. One of these problems involves only the re-scaled "masses" $\bar{\omega}_{i}$ s and the "weights" $\alpha_{i} \mathrm{~s}$ in front of them. The other problem involves, if we assume that the concentration points are not close 
pairwise or if we introduce weaker assumptions, see [6], in particular we assume that $\epsilon_{i j} \sim \frac{1}{\sqrt{\lambda_{i} \lambda_{j}}\left|a_{i}-a_{j}\right|}$, and we make further assumptions on the points $a_{i} \mathrm{~s}$, the concentrations $\lambda_{i}$ s and on the $\bar{\omega}_{i} \mathrm{~s}$, via $\bar{\omega}_{i}^{\infty}$ and $\nabla \bar{\omega}_{i}^{\infty}$. This second variational problem reads essentially:

$$
1+\bar{c}\left(---\frac{\bar{\omega}_{i}^{\infty}}{\sqrt{\lambda_{i}}}---\right)\left(\begin{array}{ccccc}
0 & \frac{-1}{\left|a_{1}-a_{2}\right|}-\frac{-1}{\left|a_{1}-a_{j}\right|}- \\
\frac{-1}{\left|a_{2}-a_{1}\right|} & 0 & -\frac{1}{\left|a_{2}-a_{j}\right|} & - \\
- & - & - & - & - \\
\frac{-1}{\left|a_{p}-a_{j}\right|} & - & - & - & 0
\end{array}\right)\left(\begin{array}{c}
- \\
- \\
\overline{\bar{\omega}_{j}^{\infty}} \\
\sqrt{\lambda_{j}} \\
- \\
-
\end{array}\right) .
$$

The additional terms, which read $-\underset{i \neq j}{\longrightarrow} \Sigma c_{i j}\left(\bar{\omega}_{i}, \bar{\omega}_{j}\right) \tilde{\epsilon}_{i j}^{3}$, may be viewed as lower order terms that can be removed under the same assumptions. $Q(V, V)$ is an additional term having $V=0$ as sole critical point $\left(|V|_{H^{1}}\right.$ is small). $Q$ is a quadratic form that has an index equal to $\underset{i=1}{\rightarrow} \stackrel{p}{\rightarrow} \Sigma\left(\operatorname{index}\left(\bar{\omega}_{i}\right)-1\right)$, where index $\left(\bar{\omega}_{i}\right)$ is the strict Morse index of $\bar{\omega}_{i}$. The matrix involved in the above expansion is denoted $A$ in the sequel.

Let us start by observing that the index of any sign-changing solution to the Yamabe problem on $\mathbb{R}^{3}$ is 2 to the least. The first variational problem described above involves the $\bar{\omega}_{i} \mathrm{~s}$ and the $\alpha_{i} \mathrm{~s}$.

Through the $\alpha_{i} \mathrm{~s}$, "masses" $\bar{\omega}_{i}$ s may be lost ie some $\alpha_{i}$ e.g. $\alpha_{1}$ becomes equal to zero along some decreasing flow-line and $\bar{\omega}_{i}$ "disappears".

Since the total variational problem is the product of two independent variational problems, a pseudo-gradient can be built that is split into two independent components near infinity. Therefore, any flow-line along which one coefficient $\alpha_{i}$, in front of some sign-changing $\bar{\omega}_{i}$, becomes zero (there may be more and, then, the requirement that they would be attached to a sign-changing $\bar{\omega}_{i}$ drops), will involve a difference of indexes larger than or equal to 2 .

\section{Driving the Morse relation at infinity}

Let us consider a Morse relation at infinity with a difference of Morse indexes equal to 1.

Either the Morse relation involves a non trivial flow-line for the first variational problem; or it involves a non trivial flow-line for the second variational problem. If the flow-line is non trivial for both, then the difference of Morse indexes is at least 2 as we will see.

If the "masses" $\bar{\omega}_{i}$ stay unchanged, but the points $\bar{a}_{i}$ change (flow-line for the second variational problem only), then the critical level at infinity is basically unchanged. The change occurs through the function $\rho$ as in [2], appropriately extended (it is an eigenvalue of the matrix $A$; the signs of the components of the corresponding eigenvector should coincide with the signs at infinity of the $\bar{\omega}_{i}\left(\bar{a}_{i}\right)$. We are assuming generic conditions as in [6]). This Morse relation at infinity is then part of a (partial) Morse complex that is a retract for the Morse relations of the full variational problem.

We therefore study here on a Morse relation where $\bar{\omega}_{1}$ for example is changed into another "mass" $\omega_{0}$ or into $\left(\Sigma \omega_{j}^{\prime}\right)_{\infty}$. In the next sub-section, we will see that Morse relations where the loss of index is precisely 1 involve precisely such and other transformations of the same type for one of the "masses" $\omega_{j}$ under some additional conditions on the Morse index of these "masses". We study here how to "drive" them so that such a transformation will lead us from a genuine critical point at infinity to another genuine critical point at infinity and not a false one. The study of the operator $\partial^{\infty}$, the intersection operator at infinity, is made easier in this way. As a preliminary step, we discuss the action of the conformal group:

The action of the Conformal Group:

Due to the action of the conformal group, $\omega_{1}$ induces a manifold of critical points. $\omega_{1}$ cannot be the standard bubble, otherwise $\bar{\omega}_{0}$ does not exist. We may then assume that this manifold is not the ball $B_{4}$.

Assume for simplicity that $\bar{\omega}_{1}$ has no symmetry. Then this manifold may be viewed as the union of two disjoint copies (two for $\pm \bar{\omega}_{1}$ ) of $S^{3} \times[1, \infty$ ). 
We break the symmetry and "compactify" (for the concentration component). $\omega_{1}$ will then induce another critical point $\hat{\omega}_{1}$ of index $(s+3)$ if $\omega_{1}$ is of (strict) index $s$. We denote $\tilde{\omega}_{1}$ the related "minimum" point in the manifold, which is of index $s$.

Assume that $\bar{\omega}_{0}$ is not the standard bubble also. We indicate below how to adjust the arguments for this later occurrence. We then break also the symmetry for $\bar{\omega}_{0}$ and we find then two critical points, $\hat{\omega}_{0}$ of index $(s+2)$ and $\tilde{\omega}_{0}$ of index $(s-1)$.

We choose $\tilde{\omega}_{1}$ and $\tilde{\omega}_{0}$ (the minima) so that their values at the North pole of $S^{3}$ (or at infinity in $\mathbb{R}^{3}$ ) are non-zero and are of the same sign. We choose $\hat{\omega}_{1}$ and $\hat{\omega}_{0}$ also so that their values at the North pole of $S^{3}$ (or at infinity in $\mathbb{R}^{3}$ ) are non-zero and are of the same sign. This will be useful in the sequel.

The Morse relations with difference of indexes equal to 1 then relate $\hat{\omega}_{1}$ to $\hat{\omega}_{0}$ and $\tilde{\omega}_{1}$ to $\tilde{\omega}_{0}$.

More masses:

When there are more "masses" and the Morse relation is therefore more complicated, the flow-line needs not connect $\tilde{\omega}_{1}$ to $\tilde{\omega}_{0}$ and $\hat{\omega}_{1}$ and $\hat{\omega}_{0}$ : this is a new flow-line, which is independent of the flow-line connecting $\tilde{\omega}_{1}$ to $\tilde{\omega}_{0}$ and $\hat{\omega}_{1}$ to $\hat{\omega}_{0}$ as it involves more "masses". The conformal group then acts on the group of $p$ masses, not on the single mass defined by $\omega_{1}$.

We present now two arguments in order to warrant that a flow-line undergoing such a transformation in the masses that it carries will connect a true critical point at infinity to another true critical point at infinity.

The first one runs as follows: after breaking the symmetry, $\hat{\omega}_{1}$ can be thought to be a bit above $\tilde{\omega}_{1}$. The same result holds for $\hat{\omega}_{0}$ and $\tilde{\omega}_{0}$.

We turn now to our critical point at infinity. The first "mass" of this critical point at infinity uses $\omega_{1}$. It therefore can be assumed to be $\tilde{\omega}_{1}$ or $\hat{\omega}_{1}$.

We also consider the critical point at infinity that it dominates. The first "mass" of this critical point at infinity uses $\omega_{0}$. It therefore can be assumed to be $\tilde{\omega}_{0}$ or $\hat{\omega}_{0}$. Since we are assuming that the difference of Morse indexes is $1, \tilde{\omega}_{1}$ will yield $\tilde{\omega}_{0}$ and $\hat{\omega}_{1}$ will yield $\hat{\omega}_{0}$.

These two couples of "masses" have the same sign at infinity (they are non-zero at infinity) and therefore, after rescaling the concentrations, we may assume that the vectors:

$$
\left(\begin{array}{c}
\frac{\bar{\omega}_{1}^{\infty}}{\sqrt{\lambda_{1}}} \\
\cdot \\
\cdot \\
\cdot \\
\frac{\bar{\omega}_{p}^{\infty}}{\sqrt{\lambda_{p}}}
\end{array}\right)=\left(\begin{array}{c}
\frac{\bar{\omega}_{0}^{\infty}}{\sqrt{\tilde{\lambda}_{1}}} \\
\frac{\omega_{2}^{\infty}}{\sqrt{\lambda_{2}}} \\
\cdot \\
\frac{\bar{\omega}_{p}^{\infty}}{\sqrt{\lambda_{p}}}
\end{array}\right)
$$

are the same. $\left(\lambda_{1}\right.$ is changed into $\tilde{\lambda}_{1}, \bar{\omega}_{2}^{\infty}, \ldots, \bar{\omega}_{p}^{\infty}$ are unchanged; $\bar{\omega}_{1}^{\infty}$ can take two values depending on whether we are considering $\tilde{\omega}_{1}$ or $\hat{\omega}_{1}$; the same holds for $\tilde{\omega}_{0}$ and $\left.\hat{\omega}_{0}\right)$.

The concentration points $\left(a_{1}, \ldots, a_{p}\right)$ are unchanged. Therefore, the two configurations are of the same nature: either they are both true critical points at infinity; or they are both false critical points at infinity. This is a remarkable fact.

We will discuss below the Morse relations that contain a flow-line between an $\omega_{1}$ and a critical point at infinity $\left(\Sigma \omega_{j}^{\prime}\right)^{\infty}$, that is configurations as above, but where $\omega_{0}$ is replaced by a critical point at infinity.

For the case when $\omega_{0}= \pm \delta_{0}$, there is the need for a more specific argument since the manifold corresponding to $\pm \delta_{0}$ is a ball (or two balls $B_{4}$ ). However, this ball appears only if there are flow-lines from $\omega_{1}$ to a constant. We may assume that such a flow-line does not take place in a more complex Morse relation, when $\omega_{1}$ has other "masses" with it $p \leq 2$, because we can assume that, when de-concentration takes place for one "mass", it takes place for all the "masses" on the flow-line that we are considering, connecting these two critical points at infinity with difference of Morse indexes equal to 1 . Therefore, the Morse relation embedded in the more complex one, 
between $\omega_{1}$ and $\pm \delta_{0}$, will fall under the arguments developed above, when the manifold was made of two copies of $S^{3} \times[A, \infty), A$ large.

Let us now present a second, more specific argument in order to analyze the Morse relations $\tilde{\omega}_{1}-\tilde{\omega}_{0}, \hat{\omega}_{1}-\hat{\omega}_{0}$ when they are embedded in more complex relations.

As already pointed out above, when there are more "masses", the flow-line needs not to connect $\tilde{\omega}_{1}$ to $\tilde{\omega}_{0}, \hat{\omega}_{1}$ to $\hat{\omega}_{0}$ : the conformal group acts on the group of $p$ masses, not on the single mass defined by $\omega_{1}$. The flow-line is originating at a critical point at infinity which is specific and contains more masses than $\omega_{1}$. We are not bound by our construction for the single $\omega_{1}$ or by the construction of the flow for another, different critical point at infinity. We can "adjust" our flow depending on the critical point at infinity that we are studying and depending on the masses that it carries. This allows us to modify the flow so that the flow-line will connect $\omega_{1}$ and $\Psi_{\lambda_{1}, \sigma}^{*} \omega_{0}$ where $\Psi_{\lambda_{1}, \sigma}^{*}$ designates the action of the conformal group, with rotation $\sigma$ (around a single axis) and concentration $\lambda_{1} . \Psi_{\lambda_{1}, \sigma}^{*} \omega_{0}$ is another element in the manifold of critical points corresponding to $\bar{\omega}_{0}$ that can be different from $\omega_{0}$.

Two occurrences may arise, see [6] for more details: let us first assume that none of the $p$ "masses" $\bar{\omega}_{i}$ is concentrated at a point $\tilde{a}_{i}$ such that $\bar{\omega}_{i}\left(\tilde{a}_{i}\right)$ is zero e.g. $\bar{\omega}_{1}$ is concentrated around a point $\tilde{a}_{1}$ where e.g $\bar{\omega}_{1}$ is positive.

In order to describe the various critical points at infinity, we can-it is in some regards a repeat of the previous argument; but we are more specific-"break" the nodal zones where $\bar{\omega}_{1}$ is positive, creating maxima for a perturbation of a functional $J$ when $\tilde{a}_{1}$ assumes some specific positions. We then restrict ourselves to one of these critical points at infinity and we complete the following construction, critical point at infinity by critical point at infinity in this family, "driving" $\bar{\omega}_{1}$ when it is concentrated around these points $\tilde{a}_{j}, j=1, \ldots, t$, to an appropriate value of $\omega_{0}, \omega_{0}^{j}$.

We choose for $\omega_{0}$ a point $b_{j}$ such that $\bar{\omega}_{0}\left(b_{j}\right)$ has the same sign than $\bar{\omega}_{1}\left(\tilde{a}_{1}\right)$ and we proceed. $\omega_{2}, \ldots, \omega_{p}$ are kept unchanged in this Morse relation. We re-scale the concentration on the first mass so that the concentration vector cited above is unchanged at the beginning and at the end of the flow-line.

We have therefore "driven" the basic Morse relation $\bar{\omega}_{1}-\bar{\omega}_{0}$ into a more complicated Morse relation when $\bar{\omega}_{1}$ is involved in a more complex critical point at infinity $\left(\omega_{1}, \ldots, \omega_{p}\right)$.

We have built the flow-lines out of $\left(\omega_{1}, \ldots, \omega_{p}\right)$ so that a flow-line $\left(\omega_{1}, \ldots, \omega_{p}-\right)-\left(\omega_{0}, \omega_{2} \ldots, \omega_{p}\right)$ corresponds to each $\bar{\omega}_{1}-\bar{\omega}_{0}$ (split into $\tilde{\omega}_{1}-\tilde{\omega}_{0}$ and $\hat{\omega}_{1}-\hat{\omega}_{0}$. We can complete the same construction for each index $i$ in $\{1, \ldots, p\}$. This takes care of the Morse relations when $\omega_{0}$ is a single mass (the mass $\delta_{0}=\omega_{0}$ is resolved as above), if $\bar{\omega}\left(\tilde{a}_{1}\right)$ is non-zero.

If $\bar{\omega}_{1}\left(\tilde{a}_{1}\right)$ is zero, then, following the Morse Lemma at infinity of [6], we may assume that all $\bar{\omega}_{j}\left(\tilde{a}_{j}\right)$ 's are zero (otherwise, a decreasing deformation can be defined, see [6]; it moves the points $\tilde{a}_{j}$ such that $\bar{\omega}_{j}\left(\tilde{a}_{j}\right)$ is zero and very close to zero, with respect to the points $\tilde{a}_{k}$ such that $\omega_{k}\left(\tilde{a}_{k}\right)$ is "far being zero".

We then "drive" the Morse relation so that $\omega_{1}$, concentrated around a zero (along its sets of zeros) is driven to $\bar{\omega}_{0}$ also concentrated around one of its zeros. we keep the relative positions and orientations of the tangent planes to $\bar{\omega}_{1}=0$ at $\tilde{a}_{1}$ and to $\omega_{0}$ at the image point $\tilde{b}_{1}$ to be the same so that the coefficients $c_{1 j}\left(\bar{\omega}_{1}, \omega_{j}\right)$ in front of $\epsilon_{i j}^{3}$, which depend on the dot product $\nabla \bar{\omega}_{1}^{\infty} \cdot \nabla \bar{\omega}_{j}^{\infty}$ is unchanged when $\bar{\omega}_{1}$ is replaced by $\bar{\omega}_{0}$. The other $c_{i j}$ s are unchanged. The concentration $\lambda_{1}$ is scaled into $\tilde{\lambda}_{1}$ so that the second factor in the expansion is unchanged.

The relation $\partial^{\infty} \circ \partial^{\infty}=0$ for the Yamabe changing-sign variational problem on bounded domains of $\mathbb{R}^{3}$

Given a bounded domain $\Omega$ of $\mathbb{R}^{3}$, we consider the problem $\Delta u+u^{5}=0$ in $\Omega, u_{\mid \partial \Omega}=0$. We assume in the sequel that this variational problem has only a finite number of solutions and we want to explore whether the relation $\partial^{\infty} \circ \partial^{\infty}=0$ holds. $\partial^{\infty}$ is the intersection operator between critical points at infinity, at infinity; that is we only count for $\partial^{\infty}$ the flow-lines staying at infinity. The other flow-lines are denoted in what follows "de-concentration flow-lines".

In the sequel, we make seven observations about the behavior of this operator and we conclude with a Conjecture/Theorem about the relation $\partial^{\infty} \circ \partial^{\infty}=0$. 
We thus consider a critical point at infinity $\underset{i=1}{\rightarrow} \stackrel{p}{\rightarrow} \sum \omega_{i, a_{i}, \lambda_{i}}, \lambda_{i}$ tending to $\infty . \omega_{i}$ rescaled is $\bar{\omega}_{i}, a_{i}$ might move in the definition of the critical point at infinity along subsets of $\Omega$, according to the unstable manifolds of the critical points of the function $\rho$ defined in Part I.

1. The first observation is the following: Assume that none of the $\omega_{i}$ s is a $\delta_{i}$. Then, along $\partial^{\infty}$, no mass $\omega_{i}$ can be lost, they are only transformed: $\omega_{i}$ into an $\omega_{i}^{\prime}$. This conclusion follows from Part II and its expansion. If a mass $\omega_{i}$, which is not $\delta_{i}$ is lost, then the loss of index is more than 1 and $\partial^{\infty}$ allows for a loss of index equal to 1 only. This is clear when the points $\left(a_{1},---, a_{p}\right)$ are "frozen" in the definition of our dominating critical point at infinity. When they are not "frozen", that is when we are considering a critical point $\left(\bar{a}_{1}, \ldots, \bar{a}_{p}\right)$ for the function $\rho\left(x_{1}, \ldots, x_{p}\right)$ with positive Morse index, then we may view our Morse relation as a combination of a loss of one or several masses $\omega_{i}$ with the projection of the unstable manifold of $\left(\bar{a}_{1}, \ldots, \bar{a}_{p}\right)$ for $\rho\left(x_{1}, \ldots, x_{p}\right)$ on the remaining dimensions corresponding to the remaining points. The sum of these dimensions cannot exceed the initial dimension of the unstable manifold of $\left(\bar{a}_{1}, \ldots, \bar{a}_{p}\right)$; therefore, any critical point at infinity further encountered along a decreasing deformation must, by the index count of Part III below, be less than the index of the dominating critical point at infinity we started from by at least 2. The claim follows.

2. The second observation is that, if two masses $\omega_{i}$ s transform, then again, reasoning as above, the loss of index is at least 2 so that the change is not along $\partial^{\infty}$ again.

3. We thus concentrate on the case when exactly one $\omega_{i}$ is changing from $\omega$ to $\omega^{\prime}$. We consider the variational problem on $S^{3}$, with the single mass $\bar{\omega}$, fully de-concentrated. It dominates $\bar{\omega}^{\prime}$ with a difference of Morse indexes equal to 1 .

Assume now that, starting from the new critical point at infinity we have reached along $\partial^{\infty}$, we have a new flow-line along $\partial^{\infty}$ and that, along this flow-line, $\omega^{\prime}$ itself changes into $\omega^{\prime \prime}$ along $\partial^{\infty}$. Then, $\bar{\omega}^{\prime}$ gives rise into $\bar{\omega} "$, with a difference of Morse indexes equal to 1 .

We consider the Morse relation $\bar{\omega}-\bar{\omega}^{\prime}-\bar{\omega}$ ". It is one branch of a kite for the changing-sign variational problem on $S^{3}$, this follows from Part I. There is another branch for the other side of this kite. If it is a branch of the type $\bar{\omega}-\bar{\omega}_{1}^{\prime}-\bar{\omega}$ ", we may re-concentrate the full kite (points are frozen here, since we have changes in the masses), getting thereby a kite for $\partial^{\infty}$, with a partial verification of the relation $\partial^{\infty} \circ \partial^{\infty}=0$. We need of course to adjust the flow-line between $\omega, \omega_{1}^{\prime}$ and $\omega^{\prime \prime}$ as above, so that the insertion of $\omega_{1}^{\prime}$ in the remainder of the dominating critical point at infinity, after removing $\omega$, is still a genuine critical point at infinity, see above ("driving the Morse relation) for the details of this process, completed in a specific way for each critical point at infinity.

If $\bar{\omega}_{1}^{\prime}$ is a critical point at infinity $\omega_{1, \infty}^{\prime}$, we need to concentrate it around a point where the sign of $\omega$ at $a_{i}$ and the sign of $\omega_{1, \infty}^{\prime}$ coincide. This can be done as soon as $\omega_{1, \infty}^{\prime}$ is not made of critical points at infinity which all $\delta_{i} \mathrm{~s}$ or $-\delta_{i} \mathrm{~s}$. Since $\omega_{1, \infty}^{\prime}$ is inserted in a critical point at infinity which dominates, along $\partial^{\infty}$, that is at infinity, a critical point at infinity where $\omega^{\prime \prime}{ }_{i}$ replaces $\omega_{1, \infty}^{\prime}$ and this is the only change, $\omega^{\prime \prime}{ }_{i}\left(a_{i}\right)$ must have the sign of $\omega\left(a_{i}\right)$ - otherwise there is an additional variation along $\rho$ and the loss of index is more than 1 - we find that $\omega_{1, \infty}^{\prime}$ can be concentrated around a point where the sign of $\omega_{1, \infty}^{\prime}$ coincides with the sign of $\omega_{i}\left(a_{i}\right)$. Again, a kite is re-formed and the relation $\partial^{\infty} \circ \partial^{\infty}=0$ is verified at the dominating critical point at infinity. The case when $\omega_{i}\left(a_{i}\right)$ is zero can be ruled out using the fact that $\left(\bar{a}_{1}, \ldots, \bar{a}_{p}\right)$ is a critical point of $\rho$. Assumptions of generic behavior have to be introduced here, in line with what we have done so far for Yamabe changing-sign problems, see [6].

4. We could also face a configuration when $\omega_{i}$ changes into $\omega_{i}^{\prime}$ and, then, at the next level, another $\omega_{i}$ changes into another $\omega_{i}^{\prime}$. Again, a kite arises after switching along the branches the order for these changes.

5. We could, instead of having a change along $\omega_{i}$, have a change of the concentration points along $\rho$. If we have two such changes along $\rho$, the dominated critical point at infinity has points of concentration $\left(\bar{b}_{1}, \ldots, \bar{b}_{p}\right)$ which form a critical point for $\rho$. We then find a kite along $\rho$.

6. We can also find a kite with a mixed branch between $\rho$ and $\omega_{i}$ changing into $\omega_{i}^{\prime}$. the same conclusion holds after careful adjustment of the points of concentration. 
7. When $\omega_{i}$ is $\delta_{a}$, we can lose $\delta_{a}$; but if the change is along $\partial^{\infty}$, the point $a$ must be "frozen". The analysis can be extended. If $\delta_{a}$ is not lost and we have a change along $\rho$ where the point $a$ moves, then $a$ must live along a manifold of dimension 1. This can be embedded into flow-lines for $\partial^{\infty}$. After taking $\partial^{\infty}$ along $\rho$, more specifically along the interval where the point $a$ is constrained to live (if it lives on an interval; it could live on a circle), $a$ is "frozen" at the next step for $\partial^{\infty}$ and the action of $\partial^{\infty}$ could correspond to a loss of $\delta_{a}$. We can again seek for the other branch of the kite and it might exist at infinity; but it could also lead us outside the critical points at infinity, since the other boundary of the interval where $a$ lives could yield a negative value for $\rho$, thereby leading the flow-lines outside of infinity.

As a conclusion, we get the following:

Conjecture/Theorem. If no $\delta_{a}$, with $a$ constrained to live on a one dimensional interval, is in the definition of a critical point at infinity, then the relation $\partial^{\infty} \circ \partial^{\infty}=0$ holds at this critical point at infinity.

The loss of one "mass" $\delta^{\infty}$ as well as the conditions that should be met for a genuine critical point at infinity is analyzed in more details in what follows:

We now analyze more specifically:

The more special case of the Morse Relations of order $1\left(\delta^{\infty}+\omega^{\infty}\right)-\omega^{\infty}$ and $\left(\omega_{1}, \ldots, \omega_{\mathrm{p}}\right)^{\infty}-\left(\left(\sum \omega_{\mathrm{j}}^{\prime}\right)^{\infty}, \omega_{2},--\right.$ ,$\left.- \omega_{\mathrm{p}}\right)^{\infty}$

Let us observe that our previous discussion extends to the case when $\omega_{0}=\left(\Sigma \omega_{j}^{\prime}\right)^{\infty}$ since we may view $\left(\Sigma \omega_{j}^{\prime}\right)^{\infty}$ as a critical point at infinity formed with a compact $\bar{\omega}_{1}^{\prime}$ to which very "concentrated other masses" $\omega_{j}^{\prime}, j \geq 2$ are added.

Of course, it is unclear whether we can think of $\left(\Sigma \omega_{j}^{\prime}\right)^{\infty}$ as a concentrated version of $\bar{\omega}_{1}^{\prime}+\left(\Sigma \omega_{j}^{\prime}\right)^{\infty}$ around a point $\tilde{a}_{1}^{\prime}$ where $\bar{\omega}_{1}^{\prime}\left(\tilde{a}_{1}^{\prime}\right)$ is non-zero and has the "right sign" as above. But again, we can "drive" the Morse relation $\omega_{1}-\left(\Sigma \omega_{j}^{\prime}\right)^{\infty}$ to be of that sort when there are more masses and the conclusion follows in this case.

We thus now consider a Morse relation $\delta^{\infty}+\omega^{\infty}-\omega^{\infty}$; we study the second factor in the expansion of the functional, after the loss of $\delta^{\infty}$ and we look at the second variational problem, with variables $\left(a_{1}, \ldots, a_{p}, \lambda_{1}, \ldots, \lambda_{p}\right)$. $a_{1}$ is gone, as well as $\lambda_{1}$.

The equation satisfied by $\left(a_{1}, \ldots, a_{p}, \lambda_{1}, \ldots, \lambda_{p}\right)$ reads, up to higher order terms, see [6] and our expansion above:

$$
\nabla_{\left(a_{1}, \ldots, a_{p}, \lambda_{1}, \ldots, \lambda_{p}\right)}\left(\frac{\bar{\omega}_{1}^{\infty}}{\sqrt{\lambda_{1}}} \ldots \frac{\bar{\omega}_{p}^{\infty}}{\sqrt{\lambda_{p}}}\right) A\left(\begin{array}{c}
\frac{\bar{\omega}_{1}^{\infty}}{\sqrt{\lambda_{1}}} \\
\cdot \\
\cdot \\
\frac{\bar{\omega}_{p}^{\infty}}{\sqrt{\lambda_{p}}}
\end{array}\right)=0
$$

subject to the constraint $\underset{i=1}{\rightarrow} \stackrel{p}{\rightarrow} \Sigma \lambda_{i}=\epsilon\left(\lambda_{i} \gtrless 0 ; \epsilon\right.$ is given positive $)$.

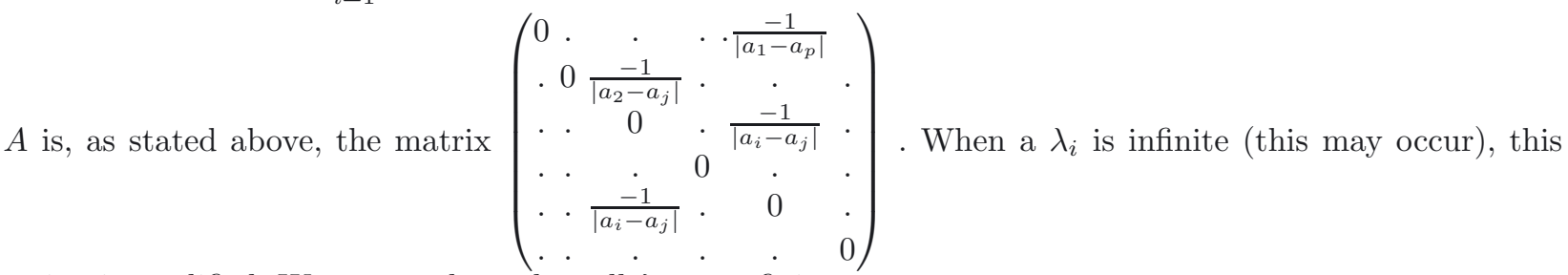
equation is modified. We assume here that all $\lambda_{i} \mathrm{~s}$ are finite.

Introducing

$$
f\left(a_{1}, \ldots, a_{p}, \lambda_{1}, \ldots, \lambda_{p}\right)=1-{ }^{t} u A u
$$


with $u=\left(\begin{array}{c}\frac{\bar{\omega}_{1}^{\infty}}{\sqrt{\lambda_{1}}} \\ \cdot \\ \cdot \\ \frac{\bar{\omega}_{p}^{\infty}}{\sqrt{\lambda_{p}}}\end{array}\right), \underset{i=1}{\rightarrow} \stackrel{p}{\rightarrow} \Sigma \frac{1}{\lambda_{i}}=\epsilon, \lambda_{i} \gtrless 0, \lambda_{i}$ can assume the value $\infty$, we find, after extending the variational theory and allowing some of the $\lambda_{i}$ s to be infinite if needed, critical points having a a definite, finite Morse index.

With $\left(a_{1}, \lambda_{1}\right)$ removed and the other points unchanged, we find a new function (similar to the previous one) with the other critical points.

There is already, with the loss of $\delta^{\infty}$, an index difference equal to 1 . With these new critical points, we should not have any additional loss of index.

A similar phenomenon arises on domains $\Omega \subset \mathbb{R}^{3}$, under Dirichlet boundary conditions, for relations of the type $\delta^{\infty}+\omega^{\infty}-\omega^{\infty}$. Clearly, if each of $a_{1}, \ldots, a_{p}$ sits, just as in [4] for example, on the top orientation class of a manifold or stratified space $M \subset \Omega, M$ of dimension 3,2 or 1 , the loss of $a_{1}$ will result in the loss of an additional index and this Morse relation will involve a difference of Morse indexes equal to 2 to the least.

A similar study should be completed for $\lambda_{1}$. We do not complete this analysis here.

There are additional conditions to be met for $\delta^{\infty}+\omega^{\infty}$ to be a genuine critical point at infinity. With $u=\left(\begin{array}{c}\frac{\bar{\omega}_{1}^{\infty}}{\sqrt{\lambda_{1}}} \\ \cdot \\ \cdot \\ \frac{\bar{\omega}_{p}^{\infty}}{\sqrt{\lambda_{p}}}\end{array}\right), \bar{\omega}_{1}=1$, we should have:

$$
{ }^{t} u A u \geq o\left(\Sigma \frac{1}{\lambda_{i}}\right)
$$

Also $\left(\bar{\omega}_{1}=1\right)$,

$$
-\left(\underset{j=2}{\rightarrow} \stackrel{\infty}{\rightarrow} \Sigma \frac{\bar{\omega}_{j}^{\infty}}{\sqrt{\lambda_{j}}\left|a_{j}-a_{1}\right|}\right) \geq o\left(\Sigma \frac{1}{\lambda_{i}}\right) .
$$

We thus see that, on $\mathbb{R}^{3}$,

$$
\left(\underset{j \neq k}{\rightarrow} \Sigma \frac{\bar{\omega}_{j}^{\infty} \bar{\omega}_{k}^{\infty}}{\sqrt{\lambda_{j} \lambda_{k}}\left|a_{j}-a_{k}\right|}\right) \leq o\left(\Sigma \frac{1}{\lambda_{i}}\right)
$$

at a critical point at infinity.

It follows that, if $\left.\underset{j=2}{\rightarrow} \stackrel{\infty}{\rightarrow} \sum \frac{\bar{\omega}_{j}^{\infty}}{\sqrt{\lambda_{j}}\left|a_{j}-a_{1}\right|}\right) \geq c\left(\Sigma \frac{1}{\lambda_{i}}\right), c \gtrless 0$, then $\omega^{\infty}$ is not a critical point at infinity since:

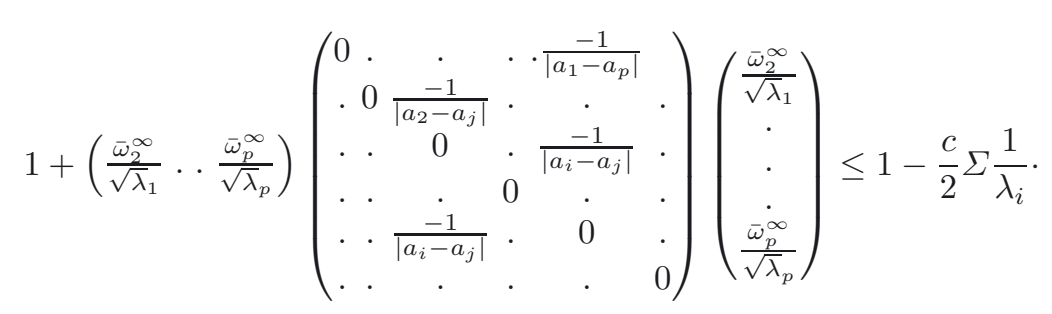

Again, under such a circumstance, there is no Morse relation of the type $\delta^{\infty}+\omega^{\infty}-\omega^{\infty}$ with a difference of Morse indexes equal to 1 . We had already seen that there was none when another type of "mass" is lost.

The conclusion of all this study is that, for several types of critical points at infinity, corresponding to special topological classes, or when the domain is $S^{3}$ or $\mathbb{R}^{3}$, there is no Morse relation entirely at infinity over the loss of "one or more masses" with a difference of Morse indexes equal to 1. 
Such Morse relations may occur even for these classes, but they involve a "de-concentration phenomenon", see $[2,4,5]$ and this holds as we deform e.g. the domains $\Omega$ under Dirichlet boundary conditions.

On the other hand, without the "loss of masses", such Morse relations, with a difference of Morse indexes equal to 1 , occur at infinity. But, they can be thought of then as simple products of a simple Morse relation, with a single mass $\omega_{1}$ as dominant term and a loss of index equal to 1 , with additional masses $\left(\omega_{2}, \ldots, \omega_{p}\right)^{\infty}$ that stay unchanged over the Morse relation.

The analysis above can be extended, with appropriate modifications, to the case when the concentrations are of various orders as they tend to infinity.

\section{Two natural questions and a conjecture}

As a consequence of the study completed above, two natural questions then arise, as well as a conjecture that helps the study of critical points at infinity on domains:

1. First Question: What is the homology $H\left(\partial^{\infty}\right)$, whenever it is defined? Is it then a retract of the homology of the full intersection operator $\partial$ for the "full" variational problem on $\Omega$ ? Due to the possible existence of "de-concentration"-flow-lines for the intersection operator $\partial$, starting at a critical point at infinity and leaving a neighborhood of "infinity", this question cannot be answered easily, even though the relation $\partial^{\infty} \circ \partial^{\infty}=0$ holds for many critical points at infinity as described above. Nevertheless, some additional (stringent) conditions may be introduced on the critical values of the variational problem at infinity on $S^{3}$ and the corresponding Morse indexes of their associated critical points at infinity so that this de-concentration process does not happen for this other branch of the kite. Whether these conditions are verified or not verified for some configurations of critical points at infinity on $S^{3}$ is an open matter which will be discussed elsewhere. Before stating the next question, we would like to sate a conjecture related to the solutions of the problem in $\Omega$ and to the critical points at infinity they give rise to. This conjecture reads as follows:

Conjecture Let $\omega$ be a changing sign solution to the Yamabe changing sign problem on $\Omega \neq S^{3}$. Then, $\left(\omega+\underset{1}{\rightarrow} \stackrel{p}{\rightarrow} \Sigma \omega_{i, a_{i}, \lambda_{i}}\right), a_{i} \in \Omega, \lambda_{i}$ tending to $\infty, a_{i} d\left(a_{i}, \partial \Omega\right)$ tending to $\infty$, is not a critical point at infinity if $p$ is large enough.

Why is this conjecture probably true?: If $\omega_{j}, a_{j}, \lambda_{j}$ has, after appropriate re-scaling, a sign at $a_{j}$ which is the sign of $\omega\left(a_{j}\right)$, then $\omega$ and $\omega_{j, a_{j}, \lambda_{j}}$ have a negative "interaction" and the functional can be decreased by decreasing $\lambda_{j}$.

Otherwise, assuming that these $\omega_{j, a_{j}, \lambda_{j}}$ are in large numbers, a large number of them is located in a nodal zone for $\omega$ where $\omega$ is e.g. positive and all the $\omega_{j, a_{j}, \lambda_{j}}$ s concentrated in this nodal zone are negative at their concentration points. We can then decrease the functional $J$ and move e.g. one of the points of concentration outside this nodal zone, into another nodal zone where the signs are opposite. A decreasing deformation, which is compact, can then be used as above.

Another natural question which arises has been known to us since we wrote [2]. It bears some similarities, within quite different frameworks, to the questions studied by Sandier and Serfaty for the Guinzburg-Landau equation. For this equation, these two authors have completed a very good work, see eg [9] for an exhaustive account of these results. It would be meaningful to extend some of their results to our present framework. The question reads as follows:

2. Second Question: "Piling up" masses $\underset{1}{\rightarrow} \stackrel{p}{\rightarrow} \Sigma \epsilon_{i} \delta_{a_{i}, \lambda_{i}}, \epsilon_{i}= \pm 1$, in various $p$ points of $\Omega$, e.g. $\Omega$ even a standard annulus, with a varying ratio $\frac{R_{e x t}}{R_{i n t}}$ and assuming that the configuration of points verifies $\rho\left(a_{1}, \ldots, a_{p}\right) \gtrless$ $0, \nabla \rho=0$, where do these masses concentrate as $p$ tends to infinity? How is the relative positions on the positive and negative masses? (they both should be "spread"). Is it possible that there exists a $p_{0}$ such that, for $p \geq p_{0}$, there are no such critical configurations of points and masses? 
In the next sub-section, we study this second question on $S^{3}$ :

\section{Configurations of points on $\mathrm{S}^{3}$}

The second variational problem:

Following our resolution of each manifold of critical points into a top and a bottom critical point that take non-zero values at the poles, the first variational problem leads us to configurations of critical points at infinity such that the relative products of their values "at infinity" $\bar{\omega}_{1}^{\infty} \times \bar{\omega}_{2}^{\infty}$ is non-zero.

Then a critical point at infinity, once the infinitely concentrated masses are removed, corresponds for the second variational problem to a critical point of an eigenvalue $\rho_{t}$ of the matrix $A\left(a_{1}, \ldots, a_{l}\right)$

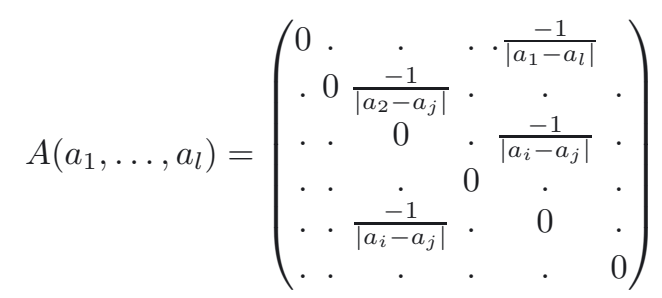

associated to the remaining points $a_{1}, \ldots, a_{l} . a_{l+1}, \ldots, a_{p}$ are additional points corresponding to masses $\pm \delta_{i}$ infinitely concentrated.

The concentration vector is $u=\left(\begin{array}{c}\frac{\bar{\omega}_{1}^{\infty}}{\sqrt{\lambda_{1}}} \\ \cdot \\ \cdot \\ \cdot \\ \frac{\bar{\omega}_{l}^{\infty}}{\sqrt{\lambda_{l}}}\end{array}\right)$.

At a critical point at infinity, the following equations are satisfied:

$$
\begin{gathered}
A u=\rho_{t} u, u \neq 0 ; \rho_{t} \geq 0 \\
\nabla_{\left(a_{1}, \ldots, a_{\ell}\right)} \rho_{t}=0 .
\end{gathered}
$$

This formula is denoted $(*)$ in the sequel. In addition, we have (a condition that generalizes to the case when $S^{3}$ or $\mathbb{R}^{3}$ are replaced with a domain $\Omega$ :

$$
\bar{\omega}_{i}^{\infty}\left(\underset{j=1}{\rightarrow} \stackrel{\ell}{\rightarrow} \Sigma \frac{\bar{\omega}_{j}^{\infty}}{\sqrt{\lambda_{j}}\left|a_{i}-a_{j}\right|}\right) \leq 0 ; i=\ell+1, \ldots, p .
$$

This formula is denoted $(* *)$ in the sequel.

$(* *)$ expresses the fact that the derivative of the expansion with respect to the concentration $\lambda_{i}, i=\ell+1, \ldots, p$ is negative. $(*)$ rereads:

$$
A u=\rho_{t} u,{ }^{t} u(\nabla A) u=0 .
$$

If we use the homogeneity of $\mathbb{R}^{3}$ or a conformal vector-field on $S^{3}$ having a zero at one of the concentration points, we find that $\rho_{t}=0$. Viewed in $\mathbb{R}^{3}$, this deformation moves the concentration points away from 0 , it might no be a compact deformation. Viewed on $S^{3}$, it is a deformation along a conformal vector-field. Using an appropriate chart, the points $a_{i}$ will move along a compact set. We thus find:

$$
(*)^{\prime} A u=0,{ }^{t} u(\nabla A) u=0 .
$$

We can create solutions for $(*)^{\prime}$ by placing equidistant masses along a meridian of $S^{3}$. This can be connected to $[8]$. 
We can also relate this equation to our earlier work [2] and we can connect it, as stated above, with the work of Sandier-Serfaty [9].

Observe that the index of a critical point at infinity defined by the masses $\left(\omega_{1}, \ldots, \omega_{p}\right)$, some under the form $\tilde{\omega}_{j}$ (group I), the other ones under the form $\hat{\omega}_{j}$ (group II), concentrated at $\left(a_{1}, . ., a_{p}\right)$, with comparable concentrations, reads as:

$$
\underset{j=1}{\rightarrow} \stackrel{p}{\rightarrow} \text { index } \bar{\omega}_{j}+3 \text { cardinal of II }+ \text { index } \rho_{t} .
$$

The term 3cardinal of II can be more generally replaced by the sum of the dimensions of the stratified subsets/submanifolds of $\Omega$ or $S^{3}$ to which the concentrations points $a_{i}$ of the "masses" of the group II belong in the definition of the critical point at infinity. Some critical points or critical points at infinity on $S^{3}$ contain such an additional variation of the concentration points $a_{i}$, see $e . g$. the construction of some solutions on $S^{3}$ due to Del Pino et al. [8]. It can also happen on domains $\Omega$ of $\mathbb{R}^{3}$.

\section{Conjectures:}

Following up on this line of ideas, we conjecture that, outside of these special configurations along meridians, the other solutions of $(*)^{\prime}$ should "spread" as the number of "masses" increases to infinity on $S^{3}$ with equal density. This should be especially true in the case of configurations of "standard bubbles $\pm \delta_{i}$. Simple arguments show that if the density on some open subset of $S^{3}$ is zero, "pushing" the "masses" in the "hole" should create a non-zero direction for $\nabla \rho$.

An interesting independent direction of research would assume that the "bubbles" are concentrated on some hypersurface of $S^{3}$ and then try to find out the constraints on the geometry (e.g. mean curvature) of the hypersurface $(*)^{\prime}$-with a number of "bubbles" tending to $\infty$ and equally spread on the hypersurface would impose on this hypersurface.

We develop in the sequel a simple argument which proves that some of these configurations cannot arise:

$(*)^{\prime}$ yields:

$$
\underset{j}{ } \Sigma \frac{\bar{\omega}_{j}}{\sqrt{\lambda_{j}}\left|a_{i}-a_{j}\right|}=0 ; i=1, \ldots, p
$$

Assuming that the $a_{i}$ s are equally spread and that to each of them is assigned a portion volume $V_{j}$, with $\frac{1}{C} \leq \frac{V_{i}}{V_{j}} \leq C$, we rewrite these identities in the form:

$$
\underset{j}{ } \Sigma \frac{\bar{\omega}_{j}}{\sqrt{\lambda_{j}} V_{j}\left|a_{i}-a_{j}\right|} \times V_{j}=0 ; i=1, \ldots, p
$$

The $\lambda_{j}$ s are also assumed to be comparable, so that $\frac{1}{C} \leq \frac{\sqrt{\lambda_{j}} V_{i}}{\sqrt{\lambda_{1}} V_{1}} \leq C$. We then set:

$$
f_{p}\left(a_{j}\right)=\frac{\sqrt{\lambda_{1}} V_{1} \bar{\omega}_{j}}{\sqrt{\lambda_{j}} V_{j}} .
$$

This means that we define a step function $f_{p}$ whose value on the ball of volume $V_{j}$ around $a_{j}$ is $f_{p}\left(a_{j}\right)$ defined above.

We also assume that, for some $\frac{4}{3} \leq q \leq 6, \underset{j}{\rightarrow} \Sigma\left(\frac{\sqrt{\lambda_{1}} V_{1}|| \omega_{j} \mid}{\sqrt{\lambda_{j}} V_{j}}\right)^{q} V_{j} \leq C$, where $C$ is a constant independent of $p$ and of the points $a_{j}$ etc. and we normalize then $f_{p}$ with a use of a constant $c$ so that $c\left(\frac{\sqrt{\lambda_{1}} V_{1}|| \omega_{j} \mid}{\sqrt{\lambda_{j}} V_{j}}\right)^{q} V_{j}=1$. We assume that the step functions $f_{p}$ converge as $p$ tends to $\infty$ to a measurable function non-zero $f$ in $L^{q}\left(R^{3}\right)$, with $|f|_{L^{q}}=1$. $(*)^{\prime}$ then yields that $\Delta^{-1} f=0$ and therefore $f=0$, a contradiction.

This indicates that such configurations verifying $(*)^{\prime}$ cannot arise with a large number of points equally spread on $S^{3}$. Of course, the "proof" that we gave of this result is only heuristic and based on several claims that would need to be established. But this indicates that the result might be true. 


\section{PART IV: Extending the SO-CAlled "BAhri-Coron" ARgument to Yamabe CHANGING SIGN-SOLUTION. A (WEAK) GENERALIZATION}

We have developed, in collaboration with Coron, a topological argument in [5] for the existence of positive solutions to Yamabe-type problems under Dirichlet boundary conditions.

There were two proofs for this argument: a geometric and intuitive proof, presented in our "Note aux ComptesRendus" [5], was our original argument. It required that no critical point of the function $\rho$ had a critical value equal to zero (this can be removed with further study). Then, another more algebraic proof was formulated with the help of Lannes [4]. It is now widely considered as the formulation of our argument, whereas the other one [5] does exist.

We would like here to thank again Lannes for his help: the second version is more compact and it can be used for other applications, which are not all about Yamabe-type equations.

We recall in the sequel the broad lines of this argument and we also develop a (weak) scheme, which requires some stringent conditions-which might nevertheless be verified, see below their specific formulation-for its application within the context of Yamabe changing-sign solutions.

The topological argument of [4] uses two fundamental facts: on one hand, the argument requires a starting point, which is the existence of a topological class $X$ which is non-zero in the e.g. $\mathbb{Z}_{2}$-homology of some lower level set for $J^{2 / 3}, J_{a}^{2 / 3}=\left\{u \in \Sigma, J^{2 / 3}(u) \leq a\right\}$.

Then, the next step is to start "piling up" "masses" $\delta(a, \lambda)$ "over" $X$, thereby moving from the level $a$ to the level $a+S$ (S the Sobolev constant), $a+2 S, \ldots, a+p S$ etc. (we are using the functional $J^{2 / 3}$ here).

At each step, as we move from $a+(p-1) S$ to $a+p S$, we construct a topological class $X_{p}$ which reads $(1-t) u+t P \delta(a, \lambda), u \in X_{p-1}, t \in[0,1] . P \delta(a, \lambda)$ is the $H_{0}^{1}$-projection of $\delta(a, \lambda)$ as in [1]. a runs in some e.g. sub-manifold of $\Omega$ which is non-zero eg $\mathbb{Z}_{2}$-homology of $\Omega$.

Then, assuming that there is no solution or critical point at infinity in between the level $a+(p-1) S$ and the level $a+p S$, the argument of [4] extends under some additional assumption: $J_{a+p S+\epsilon}^{2 / 3}$ retracts under our assumptions onto $J_{a+p S-\epsilon}^{2 / 3} \cup W$, where $W$ reads as $W=\left\{\Sigma \omega_{j, a_{j}, \lambda_{j}}+o_{H_{0}^{1}}(1) ; \epsilon_{j k}=\frac{1}{\lambda_{k} / \lambda_{j}+\lambda_{j} / \lambda_{k}+\lambda_{j} \lambda_{k} d\left(a_{j}, a_{k}\right)^{2}} \longrightarrow\right.$ $\left.0 ; \lambda_{i} d\left(a_{i}, \partial \Omega\right) \longrightarrow \infty\right\}$ if we assume, see the conjecture above, that $J^{2 / 3}$ has no critical point above the level $a$.

The level $a$ is critical at infinity for $J^{2 / 3}$. Therefore, it corresponds to a configuration $\rightarrow \stackrel{p_{0}}{\rightarrow} \sum \tilde{\omega}_{j, a_{j}, \lambda_{j}}$, where we assume that the $\omega_{j}$ s are not $\delta(a, \lambda)$-functions. Under "generic conditions (these conditions are formulated on given precise functions, thus generic conditions is an ambiguous term here), we find that $\Sigma \omega_{j, a_{j}, \lambda_{j}+o_{H_{1}^{0}}(1)}$ in $W$ reads as $\rightarrow \stackrel{p_{0}}{\rightarrow} \Sigma \tilde{\omega}_{j, a_{j}, \lambda_{j}}+\underset{1}{\rightarrow} \stackrel{s}{\rightarrow} \Sigma \delta\left(a_{k}, \lambda_{k}\right)+o_{H_{0}^{1}}(1)$ ie the additional masses are all $\delta(a, \lambda)$ s. We may then recognize the points of concentration $\left(a_{1}, \ldots, a_{s}\right)$ of these additional masses through a selection process as in $[2,4]$ and the topological argument proceeds. It can be extended in various ways.

In carrying out such an argument, we thus need first $X$. The study of $\partial^{\infty}$ completed in Part III, suitably extended with a further study of the "de-concentration" flow-lines should be helpful for finding a suitable $X$.

We then need the "generic conditions", also those needed for carrying the expansion of Part II, see [6]. This part lacks justifications, although these conditions are likely to be met.

Lastly, we need that no critical point at infinity lies between the level $a+(p-1) S$ and the level $a+p S$. This can be considerably improved: such a critical point at infinity $d^{\infty}$ must be of index equal to index $X+p+(p-1) \operatorname{dim} c$, where $c$ is the cycle in the homology of $\Omega$ which we use. In fact, some further study shows that a flow-line must exit the set $W$ at the level $a+p S$ and reach $d^{\infty}$, whereas another flow-line must exit from $d^{\infty}$ downwards, to reach the set $W$ at the level $a+(p-1) S$. Assuming that the flow-lines out of $d^{\infty}$ do not carry a de-concentration process through the function $\rho$, again this can be understood through $\partial^{\infty}$ of Part III.

The proof of these claims uses variational theory. It can be found in a paper by Cheng [7]. The idea is that the non-trivial topological class $X_{p-1}=(1-t) X_{p-2}+t \delta(a, \lambda), a \in c$, found at the level $a+(p-1) S$, 
should be "cancelled" by $d^{\infty}$. Otherwise, the argument proceeds with the addition to $J_{a+(p-1) S}^{2 / 3}$ of the unstable manifold of $d^{\infty}$. This union would then act as our new $J_{a+(p-1) S}^{2 / 3}$ and the argument would proceed.

Also, if no flow-line exits $W$ at the level $a+p S$ to reach $d^{\infty}$, then the topological class $X_{p}=(1-t) X_{p-1}+$ $t \delta(a, \lambda), t \in[0,1], a \in c$ is non-zero in $W_{u}\left(X_{p}\right) \cup J_{a+(p-1) S}^{2 / 3}$. We can add to this set $W_{u}\left(d^{\infty}\right)$. Its dimension is too low to interfere with $X_{p}$ since the dimension of $c$ is at least 1. The conclusion follows.

\section{CONCLUSION}

This set of ideas (and only partial results) is a preliminary study of the topological features for the Yamabe sign-changing variational problem on domains of $\mathbb{R}^{\nVdash}$. Some of these ideas and expansions might also be useful for the finding of non minimal solutions to the Yang-Mills equations and the harmonic map problem in dimension 2 , although the technical frameworks of these problems are different and lead to significant modifications of the techniques that have been sketched above.

Since our initial work with Coron in [1-5] etc., much progress has been completed on a wide range of variational problems belonging to the same family. This will only continue and expand.

It is a pleasure to have written this paper for the sixtieth birthday of Jean-Michel.

\section{REFERENCES}

[1] A. Bahri and J.M. Coron, On a nonlinear elliptic equation involving the critical Sobolev exponent: the effect of the topology of the domains. Commun. Pure Appl. Math. 41 (1988) 253-294.

[2] A. Bahri and J.M. Coron, Vers une Theorie des Points Critiques a l'Infini. Seminaire Bony-Sjostrand-Meyer, Expose (VIII) (1984).

[3] A. Bahri and J.M. Coron, The scalar curvature problem on the standard three-dimensional sphere. J. Funct. Anal. 95 (1991) $106-172$.

[4] A. Bahri and J.M. Coron, On a nonlinear elliptic equation involving the critical Sobolev exponent: the effect of the topology of the domain. Commun. Pure Appl. Math. 41 (1988) 253-294.

[5] A. Bahri and J.M. Coron, Sur une equation elliptique non linaire avec l'exposant critique de Sobolev. C.R. Acad. Sci. Paris 301 (1985) 345-348.

[6] A. Bahri and Y. Xu, Recent Progress in Conformal geometry. Imperial College Press, Advanced Texts in Mathematics. London (2007).

[7] Y. Chen, On a nonlinear elliptic equation involving the critical Sobolev exponent. Nonlin. Anal. 33 (1998) 41-49.

[8] M.Del Pino, M. Musso, F. Pacard and A.Pistoia, Torus action on $S^{n}$ and sign changing solutions for conformally invariant equations. Ann. Sci. Norm. Super. Pisa XII, issue 1 (2010).

[9] E. Sandier and S. Serfaty, Vortex Patterns in Ginzburg-Landau Minimizers. XVIth International Congress Om Mathematical Physics. Held 3-8 August 2009 in Prague, Czech Republic. Edited by Pavel Exner. Doppler Institute, Prague, Czech Republic. World Scientific Publishing (2010) 246-264. 